\title{
TeV gravity at neutrino telescopes
}

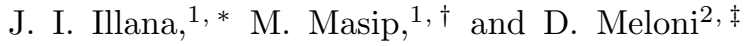 \\ 1 CAFPE and Depto. de Física Teórica y del Cosmos, Universidad de Granada, 18071 Granada, Spain \\ ${ }^{2}$ INFN and Dipto. di Fisica, Università degli Studi di Roma "La Sapienza", 00185 Rome, Italy
}

\begin{abstract}
Cosmogenic neutrinos reach the Earth with energies around $10^{9} \mathrm{GeV}$, and their interactions with matter will be measured in upcoming experiments (Auger, IceCube). Models with extra dimensions and the fundamental scale at the TeV could imply signals in these experiments. In particular, the production of microscopic black holes by cosmogenic neutrinos has been extensively studied in the literature. Here we make a complete analysis of gravity-mediated interactions at larger distances, where they can be calculated in the eikonal approximation. In these processes a neutrino of energy $E_{\nu}$ interacts elastically with a parton inside a nucleon, loses a small fraction $y$ of its energy, and starts a hadronic shower of energy $y E_{\nu} \ll E_{\nu}$. We analyze the ultraviolet dependence and the relevance of graviton emission in these processes, and show that they are negligible. We also study the energy distribution of cosmogenic events in AMANDA and IceCube and the possibility of multiple-bang events. For any neutrino flux, the observation of an enhanced rate of neutral current events above $100 \mathrm{TeV}$ in neutrino telescopes could be explained by TeV-gravity interactions. The values of the fundamental scale of gravity that IceCube could reach are comparable to those to be explored at the LHC.
\end{abstract}

PACS numbers: 04.50.+h, 13.15.+g, 96.40.Tv

\section{INTRODUCTION}

Cosmogenic neutrinos appear in any scenario proposed to explain the most energetic cosmic rays. In particular, if the observed air showers of up to $10^{11} \mathrm{GeV}$ [1] are produced by primary protons, in their way to the Earth these protons will interact with the cosmic microwave background $(\mathrm{CMB})$ photons and produce pions:

$$
p+\gamma_{2.7 \mathrm{~K}} \rightarrow \Delta^{+} \rightarrow n+\pi^{+}\left(p+\pi^{0}\right) .
$$

The flux of cosmogenic neutrinos would then be created in the decay of the charged pions, and it will appear correlated with observable fluxes of nucleons and photons (see 2] for a recent review).

Cosmogenic neutrinos are of great interest as probes of new $\mathrm{TeV}$ physics because they provide very large center of mass energies. In addition, the relative effect of new physics on the weakly interacting neutrinos is larger than on quarks or charged leptons, making it easier to see deviations. At these energies the new physics may be able to compete with the weak interactions and provide signatures that could be detected in deeply penetrating air showers and neutrino telescopes.

In particular, one expects that at transplanckian energies gravity dominates over all the other interactions. This will be the case when a cosmogenic neutrino interacts with a terrestrial nucleon in models with extra dimensions and the fundamental scale $M_{D}$ at the TeV [3] . The possibility of black hole (BH) formation [4] by cosmogenic neutrinos has been discussed by several groups

\footnotetext{
*Electronic address: jillana@ugr.es

$\dagger$ Electronic address: masip@ugr.es

‡Electronic address: meloni@roma1.infn.it
}

[5, 6, 7, 8, 9, 10, 11]. These analyses are based on a geometric cross section, which assumes gravitational collapse if the neutrino interacts at impact parameter distances smaller than the Schwarzschild radius $R_{S}$ of the system. The collapse involves strongly coupled gravity and is not calculable perturbatively, but if $R_{S} \gg M_{D}^{-1}\left(\sqrt{s} \gg M_{D}\right)$ one expects that the estimate will not be off by any large factors [10]. It is found, however, that the $\nu N$ cross section is dominated by the low- $x$ region, with $\sqrt{s}$ at the parton level close to $M_{D}$, and most of the BHs produced will have a radius $R_{S} \sim M_{D}^{-1}$. In this regime the amount of gravitational radiation emitted during the collapse or the topology of the singularity are important effects that add uncertainty to the geometric estimate.

Here we study the gravitational interaction at larger distances, where it can be calculated using the eikonal approximation [11, 12, 13, 14]. This approximation involves linearized gravity and is not affected by the uncertainties in the cross section for $\mathrm{BH}$ formation. In the next Section we show that, for the typical energy $E_{\nu}$ of cosmogenic neutrinos, the eikonalized $\nu N$ cross section depends very mildly on how the theory is completed in the ultraviolet (UV), at energies around $M_{D}$. We also show that the amount of gravitational radiation emitted during the scattering is small. In these processes the neutrino interacts at impact parameters larger than $R_{S}$ and therefore with a larger cross section than for $\mathrm{BH}$ production. At the parton level the neutrino scatters elastically and transfers a small fraction $y$ of its energy to a quark or a gluon, which starts then a hadronic shower of energy $y E_{\nu}$. Three main features characterize these processes and distinguish them from standard model or $\mathrm{BH}$ events. First, the shower has a typical energy much smaller than the energy $\left(10^{8}\right.$ to $\left.10^{11} \mathrm{GeV}\right)$ of the incoming neutrino. Second, a charged lepton is never produced in the starting point of the shower. Finally, the neutrino 
is not destroyed in the interaction, it keeps going with essentially the same energy and may interact again. We show in Section III that neutrino telescopes are then ideal experiments to observe these elastic processes: they are designed to detect hadronic showers of energy down to $100 \mathrm{TeV} \ll 10^{9} \mathrm{GeV}$ (below $100 \mathrm{TeV}$ the atmospheric background dominates), and their big volume $\left(1 \mathrm{~km}^{3}\right.$ in IceCube [15]) would favor multiple-bang events. In the final section we discuss and summarize our results. Our analysis here completes our work in 12], where aspects like the UV dependence of the eikonal amplitude, gravitational bremsstrahlung, or multiple-bang events in neutrino telescopes were not discussed.

\section{TEV GRAVITY}

The simplest picture of $\mathrm{TeV}$ gravity includes only two free parameters: the value of the higher-dimensional Planck scale $M_{D}$, and the number $n$ of compact dimensions where gravity propagates. A third parameter, the (common) length $2 \pi R$ of the $n$ dimensions, could be deduced from the 4-dimensional Newton constant $G_{N} \equiv M_{P}^{-2}$ :

$$
G_{D}=(2 \pi R)^{n} G_{N}=\frac{(2 \pi)^{n-1}}{4 M_{D}^{n+2}} .
$$

At processes below $M_{D}$ the model-independent signature of extra dimensions is graviton emission. The amount of energy radiated would be proportional to the accessible phase space or, in the Kaluza-Klein (KK) picture, to the number of KK modes of mass below the center of mass energy. In this type of experiments for a given $n$ one sets bounds on $R$ and then deduces the limits on $M_{D}$. From collider experiments one obtains $M_{D} \geq 1.4$ (1.0) TeV for $n=2(\geq 3)$ [16], whereas from SN1987A the bounds go up to $22 \mathrm{TeV}$ for $n=2$ [17]. One should keep in mind, however, that the gravitons emitted in the supernova explosion have a KK mass below $\approx 50 \mathrm{MeV}$. The simple picture with two extra flat dimensions could be modified above $50 \mathrm{MeV}$, for example, with four more dimensions at $R^{\prime} \sim(100 \mathrm{GeV})^{-1}$, which would bring the fundamental scale of gravity down to 1 $\mathrm{TeV}$ without affecting the physics in the supernova. It could also be that some other mechanism (a warp factor in [18]) gives an extra mass of order $\geq 50 \mathrm{MeV}$ to the KK excitations, invalidating all the bounds based on supernovas.

The bounds obtained from transplanckian collisions are complementary in the sense that given $n$ they are a direct probe of $M_{D}$, and $R$ is then adjusted in order to reproduce $G_{N}$. At energies above $M_{D}$ and impact parameters smaller than $R$ the collision is a pure higherdimensional process independent of the compactification details that fix the value of the effective Newton constant. The transplanckian collision does not see that the extra dimensions are compact, they could be taken infinite with no effect on the cross section.

\section{A. Neutrino-parton amplitude}

The $\mathrm{TeV}$ gravity model should be embedded in a string theory, which would relate $M_{D}$ with the string scale $M_{S}$. In the simplest set-up [19] the standard model (SM) fields (open strings) would be attached to a 4-dimensional brane, whereas gravity (closed strings) would propagate in the whole $D$-dimensional space. In this case

$$
M_{D}^{n+2}=\frac{8 \pi}{g^{4}} M_{S}^{n+2},
$$

with $g$ the string coupling. The transplanckian regime corresponds then to energies above the string scale, where any tree-level amplitude becomes very soft. In the ultraviolet string amplitudes go to zero exponentially at fixed angle and, basically, only the forward (long-distance) contribution of the graviton survives (the forward contribution of the SM gauge bosons also survives, but it is subleading above $M_{D}$ due to the smaller spin of the vector bosons). This is precisely the regime where the eikonal approximation is valid.

Let us consider the elastic collision of a neutrino and a parton that exchange $D$-dimensional gravitons (see 11 , 14] for details). The eikonal amplitude $\mathcal{A}_{\text {eik }}(s, t)$ resums the infinite set of ladder and cross-ladder diagrams. It is reliable as far as the momentum carried by the gravitons is smaller than the center of mass energy or, in terms of the fraction of energy $y=\left(E_{\nu}-E_{\nu}^{\prime}\right) / E_{\nu}$ lost by the incoming neutrino, if $y=-t / s \ll 1(s$ and $t$ refer to the Mandelstam parameters at the parton level). In this limit the amplitude is independent of the spin of the colliding particles. Essentially, $\mathcal{A}_{\text {eik }}$ is the exponentiation of the Born amplitude in impact parameter space:

$$
\mathcal{A}_{\mathrm{eik}}(s, t)=\frac{2 s}{i} \int \mathrm{d}^{2} b e^{i \mathbf{q} \cdot \mathbf{b}}\left(e^{i \chi(s, b)}-1\right),
$$

where $\chi(s, b)$ is the eikonal phase and $\mathbf{b}$ spans the (bidimensional) impact parameter space. The Born amplitude corresponds to $\mathcal{A}_{\text {eik }}(s, t)$ in the limit of small $\chi(s, b)$ and, therefore, the eikonal phase can be deduced from the Fourier transform to impact parameter space of $\mathcal{A}_{\text {Born }}(s, t)$ :

$$
\chi(s, b)=\frac{1}{2 s} \int \frac{d^{2} q}{(2 \pi)^{2}} \mathrm{e}^{-i \mathbf{q} \cdot \mathbf{b}} \mathcal{A}_{\text {Born }}\left(s, q^{2}\right) .
$$

Our Born amplitude comes from the $t$-channel exchange of a higher-dimensional graviton:

$$
\mathcal{A}_{\text {Born }}=-\frac{s^{2}}{M_{D}^{n+2}} \int \frac{\mathrm{d}^{n} q_{T}}{t-q_{T}^{2}},
$$

where the integral over momentum $q_{T}$ along the extra dimensions (equivalent to the sum over KK modes) gives an UV divergence if $n \geq 2$. The magic of the eikonal amplitude is that it will be well defined despite we obtain it from an UV dependent Born amplitude. To understand that, let us first evaluate $\chi(s, b)$ using dimensional 


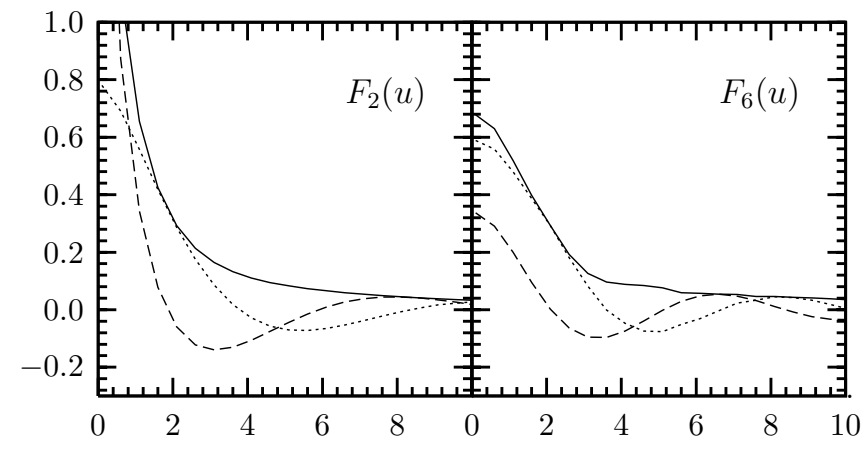

FIG. 1: The real (dashed) and imaginary (dotted) parts and the moduli (solid) of the functions $F_{n}$ in the eikonal amplitude (9) for $n=2,6$.

regularization. The Born amplitude becomes

$$
\mathcal{A}_{\mathrm{Born}}(s, t)=\frac{s^{2}}{M_{D}^{n+2}} \pi^{\frac{n}{2}}(-t)^{\frac{n}{2}-1} \Gamma\left(1-\frac{n}{2}\right),
$$

which implies

$$
\begin{aligned}
\chi(s, b) & =\frac{\pi^{\frac{n}{2}-1} \Gamma\left(1-\frac{n}{2}\right) s}{4 M_{D}^{n+2}} \int_{0}^{\infty} d q q^{n-1} J_{0}(q b) \\
& =\frac{1}{b^{n}} \frac{(4 \pi)^{\frac{n}{2}-1}}{2} \Gamma\left(\frac{n}{2}\right) \frac{s}{M_{D}^{n+2}} \equiv\left(\frac{b_{c}}{b}\right)^{n} .
\end{aligned}
$$

Although the eikonal phase diverges at $b=0$, the amplitude in Eq. (4) is insensitive to that: the contributions from the region $b \ll b_{c}$ are quickly oscillating and tend to cancel. $\mathcal{A}_{\text {eik }}(s, q)$ can be written

$$
\mathcal{A}_{\mathrm{eik}}(s, q)=4 \pi s b_{c}^{2} F_{n}\left(b_{c} q\right),
$$

with

$$
F_{n}(u)=-i \int_{0}^{\infty} \mathrm{d} v v J_{0}(u v)\left(e^{i v^{-n}}-1\right)
$$

where $q=\sqrt{-t}$, and the integration variable is $v=b / b_{c}$ (see Fig (1). For $q<b_{c}^{-1}$ this integral is dominated by impact parameters around $b_{c}$, and for $q>b_{c}^{-1}$ by a saddle point at $b_{s}=b_{c}\left(n / q b_{c}\right)^{(1 / n+1)}$.

\section{B. Cutoff dependence of the amplitude}

Let us now use an UV cutoff $\Lambda$ to regularize the Born amplitude in Eq. (6). This may be more physical than dimensional regularization since $D$-dimensional gravity must be completed (embedded in a consistent theory) at energies around $M_{D}$. For example, in the simple braneworld setting mentioned above all the KK excitations of the graviton with mass (or $q_{T}^{2}$ ) larger than the string scale decouple exponentially [20], an effect that would mimic a cutoff. In any case, we can use the cutoff to estimate the UV dependence of these eikonalized processes.
The Born amplitude becomes

$$
\begin{aligned}
\mathcal{A}_{\text {Born }}(s, q) & =\frac{s^{2}}{M_{D}^{n+2}} \frac{2 \pi^{n / 2}}{\Gamma\left(\frac{n}{2}\right)} \int_{0}^{\Lambda} d q_{T} \frac{q_{T}^{n-1}}{q_{T}^{2}+q^{2}} \\
& =\frac{s^{2}}{M_{D}^{n+2}} \frac{2 \pi^{n / 2}}{\Gamma(n / 2)} q^{n-2} I_{n}(\Lambda / q),
\end{aligned}
$$

where $I_{n}(\Lambda / q)$ diverges like $(\Lambda / q)^{n-2}$ with the cutoff (the divergence is logarithmic for $n=2)$. It is now straightforward to find

$$
\chi(s, b)=\frac{\pi^{\frac{n}{2}-1} s}{2 M_{D}^{n+2} \Gamma(n / 2)} \frac{1}{b^{n}} \int_{0}^{\Lambda b} d \xi \xi^{n-1} K_{0}(\xi)
$$

where $K_{0}(\xi)$ is a modified Bessel function of the second kind. Expanding (12) in powers of $\Lambda b$ we obtain

$$
\chi(s, b)=\left(\frac{b_{c}}{b}\right)^{n}\left[1-\sqrt{\frac{\pi}{2 \Lambda b}} \mathrm{e}^{-\Lambda b} A_{n}(\Lambda b)\right],
$$

with $A_{n}(\Lambda b)$ approaching the constant $2^{2-n} / \Gamma^{2}(n / 2)$ for large values of $\Lambda b$. This expression tells us that the cutoff introduces corrections to the eikonal phase which are relevant only at impact parameters $b \leq \Lambda^{-1}$. This region in $b$ gives a negligible contribution to $\mathcal{A}_{\text {eik }}(s, q)$ (see Section (ID).

\section{Non-linear corrections and soft graviton emission}

The eikonal amplitude in Eq. (9) is well defined for all values of $s$ and $q$. However, as $q$ (or $y=q^{2} / s$ ) grows nonlinear corrections ( $\mathrm{H}$ diagrams) become important [14]. The relevance of $\mathrm{H}$ diagrams implies a regime with strong gravitational coupling and important graviton emission (soft bremsstrahlung). The strong coupling can be expected just by inspecting the eikonal amplitude, since for $-t / s \approx 1$ the saddle point $b_{s}$ that dominates the integral in impact parameter space approaches the Schwarzschild radius $R_{S}$ [1] of the system:

$$
R_{S}=\left[\frac{2^{n} \pi^{\frac{n-3}{2}} \Gamma\left(\frac{n+3}{2}\right)}{n+2}\right]^{\frac{1}{n+1}}\left(\frac{s}{M_{D}^{2 n+4}}\right)^{\frac{1}{2(n+1)}} .
$$

A process with typical impact parameter $b \leq R_{S}$ will not be properly described by the eikonal amplitude, since nonlinear corrections will be of order one. On the other hand, in eikonal processes with $y \ll 1$ the main contribution to the amplitude in Eq. (4) comes from impact parameters much larger than $R_{S}$, where nonlinear effects are small (see Section ID).

Soft graviton emission is also a consequence of nonlinear couplings, it appears as an imaginary contribution to the eikonal phase corrected by $\mathrm{H}$ diagrams $\left(\chi_{H}\right)$ 21]. This contribution is of absorptive type, it damps the elastic cross section showing that a Bloch-Nordsieck mechanism is at work. For a given value of $b$, the average 
number $N_{\text {soft }}$ of gravitons radiated during the scattering can be read directly from $\chi_{H}$ [14, 21]:

$$
N_{\text {soft }}=\operatorname{Im}\left(\chi_{H}\right) \approx\left(\frac{b_{r}}{b}\right)^{3 n+2},
$$

where

$$
b_{r} \equiv\left(b_{c}^{n} R_{S}^{2 n+2}\right)^{\frac{1}{3 n+2}} \approx\left(G_{D}^{3} s^{2}\right)^{\frac{1}{3 n+2}} .
$$

Therefore, the typical (transverse) momentum radiated will be $Q \approx N_{\text {soft }} b^{-1}$. Notice that to obtain the energy lost by the incoming neutrino this momentum must be boosted to the nucleon rest frame. In an eikonal scattering the dominant impact parameter distance is $\langle b\rangle \approx b_{s}$. Both $Q \approx b_{s}^{-1} \approx M_{D}\left(y M_{D}^{2} / s\right)^{1 / 2 n+2}$ and the number of gravitons $N_{\text {soft }} \approx y^{(3 n+2) /(2 n+2)}\left(s / M_{D}^{2}\right)^{(n+2) /(2 n+2)}$ decrease for decreasing values of $y$, implying that for $y \ll 1$ the amount of gravitational radiation during the scattering is small (see below a numerical example).

On the other hand, in a collision at $\langle b\rangle \approx R_{S}$ one expects a large fraction of energy transferred from the neutrino to the parton, a large scattering angle, and a significant fraction of energy lost to radiation. At these and smaller values of $b$ one would also expect black hole $(\mathrm{BH})$ formation [4, 5, 6, 7, 8, 9, 10, 11]. It has been shown, however, that a number of factors (angular momentum, charge, geometry of the trapped surface, radiation before the collapse) make a precise estimate difficult, specially for light BHs of mass just above $M_{D}$.

\section{Numerical analysis of the $\nu N$ eikonal cross section}

To understand the relative relevance of the different scales and processes involved, in this Section we will consider the scattering of a $10^{10} \mathrm{GeV}$ neutrino with a nucleon $N=(n+p) / 2$. The $\nu N$ center of mass energy is in this case $\sqrt{s}=\sqrt{2 m_{N} E_{\nu}}=141 \mathrm{TeV}$. We will take $n=2$ or $n=6$ extra dimensions and a fundamental scale $M_{D}=1 \mathrm{TeV}$. The transplanckian regime will then include the partonic processes of energy $\sqrt{\hat{s}}=\sqrt{x s}>M_{D}$, i.e., $x>5 \times 10^{-5}$. To evaluate the cross sections we will use the CTEQ5 parton distribution functions (PDFs) 22], which are available both for fortran and Mathematica codes. We will base our analysis on the kinematical variable $y=\left(E_{\nu}-E_{\nu}^{\prime}\right) / E_{\nu}$, which fixes $q^{2}=y \hat{s}$ and the dominant impact parameter distance $\langle b\rangle$ in the eikonal process $\left(\langle b\rangle \approx b_{s}\right.$ if $q>b_{c}^{-1}$ or $\langle b\rangle \approx b_{c}$ if $\left.q<b_{c}^{-1}\right)$. We will evaluate the PDFs at this dominant distance $\left(\mu=\langle b\rangle^{-1}\right)$.

We find (see Fig. 2) that the differential cross section

$$
\frac{d \sigma_{\text {eik }}^{\nu N}}{d y}=\int_{M_{D}^{2} / s}^{1} d x x s \pi b_{c}^{4}\left|F_{n}\left(b_{c} q\right)\right|^{2} \sum_{i=q, \bar{q}, g} f_{i}(x, \mu)
$$

grows as $y$ decreases 11]. For example, for $n=2(6)$ it is a factor of $265(62)$ larger at $y=10^{-3}$ than at $y=0.1$.

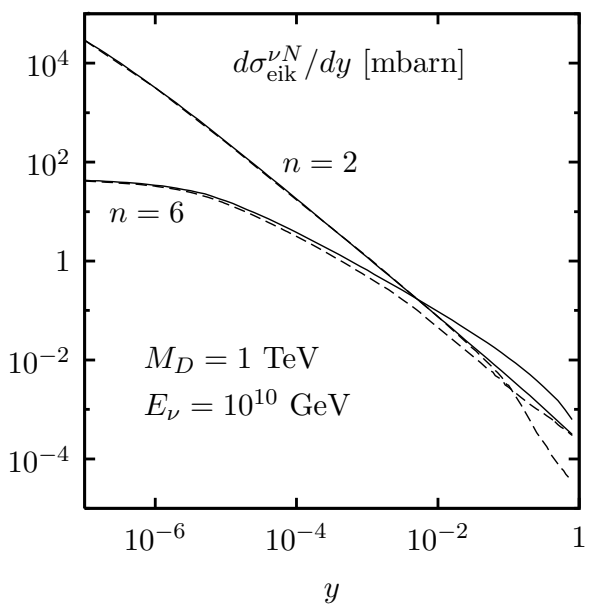

FIG. 2: Differential cross section $\mathrm{d} \sigma_{\text {eik }}^{\nu N} / \mathrm{d} y$ of a $10^{10} \mathrm{GeV}$ neutrino for $n=2,6$ and an UV cutoff $\Lambda \rightarrow \infty$ (solid) and $\Lambda=M_{D}$ (dashed).

The small $y$ region corresponds to long distance processes where the neutrino interacts with a parton and transfers only a small fraction of its energy. This region is less important for $n=6$ than for $n=2$ extra dimensions, since then gravity dilutes faster and becomes weaker at long distances. On the other hand, values of $y$ close to 1 mean shorter distance interactions. Using Eq. (9) we can evaluate the contribution to the cross section from different regions in impact parameter space. We obtain, for example, that for a $\nu N$ process with $y=0.5$ a $52 \%$ of the eikonal cross section comes from impact parameters $b<R_{S}$ if $n=2$ (or a $71 \%$ if $n=6$ ). In these processes with $y \approx 1$ the eikonal amplitude will be corrected by nonlinear contributions of the same order. Therefore, we will use the eikonal amplitude to evaluate elastic processes with $y<y_{\max } \approx 0.2$ only. Our results will depend very mildly on the actual value of $y_{\max }$, since the bulk of the cross section comes from the small $y$ region. For example, for $n=2$ the eikonal $\nu N$ cross section for processes with $10^{-6} \leq y \leq y_{\max }$ is $\sigma_{\text {eik }}^{\nu N}=1.97 \times 10^{-2} \mathrm{mb}$ if $y_{\max }=0.4$ or $\sigma_{\text {eik }}^{\nu N}=1.91 \times 10^{-2} \mathrm{mb}$ if $y_{\max }=0.1$. For $n=6$ the values of the cross would change with $y_{\max }$ from $7.5 \times 10^{-3} \mathrm{mb}$ to $6.2 \times 10^{-3} \mathrm{mb}$.

The numerical relevance of the UV cutoff (see Section III) is expressed in Fig. 2] where we plot $\mathrm{d} \sigma_{\text {eik }}^{\nu N} / \mathrm{d} y$ for $\Lambda \rightarrow \infty$ (solid lines) and for $\Lambda=M_{D}$ (dashed line). In the later case KK modes of the graviton heavier than $M_{D}$ are decoupled. We find that the $\nu N$ differential cross section changes less than a $10 \%$ for $10^{-5}<y<10^{-2}$, so the cutoff dependence of the eikonal amplitude in these processes is not important.

Let us now consider the geometric cross section (at the parton level) $\sigma_{\mathrm{BH}}=\pi R_{S}^{2}$, with $R_{S}$ given in Eq. (14). $\sigma_{\mathrm{BH}}$ includes all the processes (elastic and inelastic) at impact parameter distances $b$ below $R_{S}$, and it can be used to estimate the rate of black hole formation. As explained before, eikonal scatterings of small $y$ will be dominated 
by values of $b$ larger than $R_{S}$. Therefore, the overlapping between these soft eikonal processes and the processes in the (inclusive) geometrical cross section will be negligible. It is then justified to consider two types of transplanckian $\left(\hat{s}>M_{D}^{2}\right)$ processes: elastic (long-distance) processes where the neutrino transfers to the partons a small fraction $y<y_{\max }$ of its energy and keeps going, and shorter distance $\left(b<R_{S}\right)$ hard processes where the neutrino loses in the collision most of its energy, possibly collapsing into a BH. To estimate the relative frequency of these two processes when a $10^{10} \mathrm{GeV}$ neutrino scatters off a nucleon, we can compare the eikonal cross section $\sigma_{\text {eik }}^{\nu N}$ with $y$ integrated between $10^{-5}$ and 0.2 with $\sigma_{\mathrm{BH}}^{\nu N}$. For $n=2(6)$ we obtain $\sigma_{\text {eik }}^{\nu N}=1.94 \times 10^{-2} \mathrm{mb}\left(6.88 \times 10^{-2} \mathrm{mb}\right)$ and $\sigma_{\mathrm{BH}}^{\nu N}=9.82 \times 10^{-4} \mathrm{mb}\left(4.07 \times 10^{-3} \mathrm{mb}\right)$, i.e., the neutrino will have 12.5 (1.64) interactions in which it transfers to the nucleon between $100 \mathrm{TeV}$ and $2 \times 10^{9} \mathrm{GeV}$ of energy, per each short distance (black hole) interaction. The total energy lost by the neutrino in these 12.5 (1.64) interactions is

$$
\begin{aligned}
E_{\text {eik }} & =\frac{1}{\sigma_{\mathrm{BH}}^{\nu N}} \int_{\frac{100 \mathrm{TeV}}{E_{\nu}}}^{y_{\max }} d y y E_{\nu} \frac{d \sigma_{\text {eik }}^{\nu N}}{d y} \\
& =1.00 \times 10^{9} \mathrm{GeV}\left(5.04 \times 10^{8} \mathrm{GeV}\right) .
\end{aligned}
$$

Finally, let us comment on the amount of gravitational energy radiated in these eikonalized scatterings. In a parton process of energy $\sqrt{x s}$ and inelasticity $y$ the energy lost to radiation in the c.o.m. frame is

$$
E_{\text {rad }}^{*}(x, y) \approx \min \left\{\frac{1}{\langle b\rangle}\left(\frac{b_{r}}{\langle b\rangle}\right)^{3 n+2}, \sqrt{x s}\right\},
$$

which in the nucleon at rest frame is

$$
E_{\mathrm{rad}}(x, y)=E_{\mathrm{rad}}^{*} \sqrt{\frac{E_{\nu}}{2 x m_{N}}} .
$$

The average energy lost through soft bremsstrahlung per each short distance gravitational interaction is then

$$
\left\langle E_{\mathrm{rad}}\right\rangle=\frac{1}{\sigma_{\mathrm{BH}}^{\nu N}} \int_{M_{D}^{2} / s}^{1} d x \int_{0}^{y_{\mathrm{max}}} d y E_{\mathrm{rad}} \frac{d^{2} \sigma_{\text {eik }}^{\nu N}}{d x d y}
$$

We find that, for $n=2(6)$, during the 12.5 (1.64) eikonal processes the $10^{10} \mathrm{GeV}$ neutrino radiates soft gravitons with a total energy of $1.56 \times 10^{9} \mathrm{GeV}\left(4.90 \times 10^{8} \mathrm{GeV}\right)$.

\section{SIGNALS AT NEUTRINO TELESCOPES}

The flux of cosmogenic neutrinos depends on the production rate of primary nucleons of energy around and above the GZK cutoff $E_{\mathrm{GZK}}$. It will always appear correlated with proton and photon fluxes that should be consistent, respectively, with the number of ultrahigh energy events at AGASA and HiRes [1] and with the diffuse $\gamma$ ray background measured by EGRET [23].

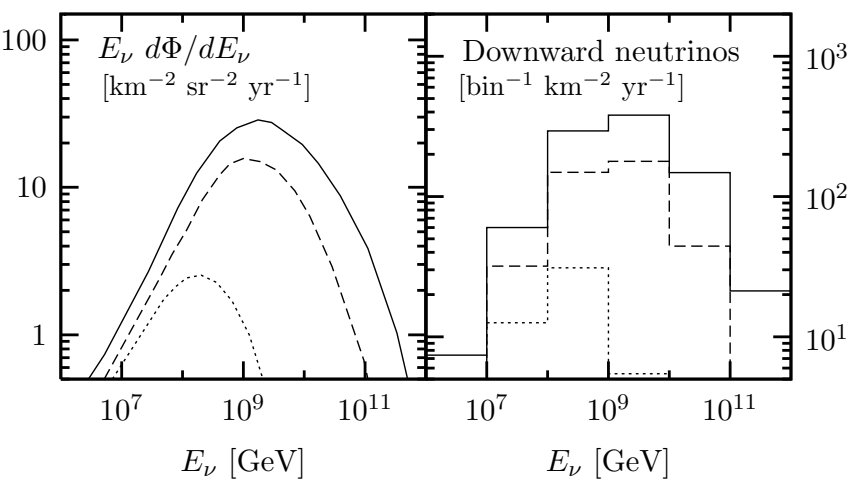

FIG. 3: Left: cosmogenic neutrino fluxes referred in the text as higher (solid), lower (dashed) and minimal (dotted). We plot the fluxes for one flavor $\Phi=\phi_{\nu_{\ell}}+\phi_{\bar{\nu}_{\ell}}$ and assume that all flavors have the same frequency. Right: corresponding number of downward $\left(0 \leq \theta_{z}<\pi / 2\right)$ cosmogenic neutrinos of each flavor.

We will base our analysis on the two neutrino fluxes described in 2] (solid and dashed lines in Fig. 31). The first one saturates the observations by EGRET, whereas for the second one the correlated flux of $\gamma$-rays contribute only a $20 \%$ to the data, with the nucleon flux normalized in both cases to AGASA/HiRes. The higher flux predicts 820 downward neutrinos of each flavor with energy between $10^{8} \mathrm{GeV}$ and $10^{11} \mathrm{GeV}$ per year and $\mathrm{km}^{2}$, versus 370 for the lower one. We will also comment on the minimal flux described in 24] (dots in Fig. 3), where the cosmic ray events above the GZK cutoff are assumed not to be protons [25]. The proton events around and below $E_{\mathrm{GZK}}$ imply then just 35 downward neutrinos of each flavor with energy between $10^{8} \mathrm{GeV}$ and $10^{11} \mathrm{GeV}$ per year and $\mathrm{km}^{2}$.

To observe a hadronic event inside the telescope, a cosmogenic neutrino must first survive as it crosses the atmosphere and the ice (or water) above the detector. Its typical interaction length in a medium of density $\rho$ is

$$
L_{0}=\frac{1}{\rho N_{A} \sigma^{\nu N}},
$$

where $\sigma^{\nu N}=\sigma_{\mathrm{SM}}^{\nu N}+\sigma_{\mathrm{BH}}^{\nu N}$ is the total cross section to have an interaction that destroys the neutrino. It is usual to express the length in terms of its depth: $x_{0}=\rho L_{0}$ (i.e. one meter of water has a depth of $100 \mathrm{~g} / \mathrm{cm}^{2}$ ). Notice that we include in $\sigma^{\nu N}$ both the SM and the short distance gravitational interactions, but we ignore the soft (eikonalized) gravitational interactions because they take from the neutrino just a small fraction of its energy (the distortion in the flux of neutrinos that reach the detector that these interactions produce is negligible).

A neutrino from a zenith angle $\theta_{z}$ must cross a column density of material

$$
x\left(\theta_{z}\right)=\int_{\theta_{z}} d l \rho\left(l, \theta_{z}\right) .
$$


In practice, the path in the atmosphere is negligible and $x\left(\theta_{z}\right)$ is just the depth of the water or ice above the detector. The probability that it does not interact before reaching the detector is then

$$
P_{\text {surv }}\left(E_{\nu}, \theta_{z}\right)=\mathrm{e}^{-x / x_{0}} .
$$

Once in the detector, the probability of an event is

$$
P_{\text {int }}\left(E_{\nu}\right) \approx 1-\mathrm{e}^{-L \rho N_{A} \sigma_{\text {int }}^{\nu N}},
$$

where $L$ is the linear dimension of the detector and $\sigma_{\text {int }}^{\nu N}$ the total cross section. Therefore, the total number of events in the telescope in an observation time $T$ is

$$
N=2 \pi A T \int d E_{\nu} \sum_{\nu_{i}, \bar{\nu}_{i}} \frac{d \phi_{\nu_{i}}}{d E_{\nu}} \int d \cos \theta_{z} P_{\text {surv }} P_{\text {int }}
$$

where $A$ is the detector's cross sectional area and $\phi_{\nu_{i}}$ the neutrino flux. Before a complete numerical analysis we would like to discuss the possibility of multiple-bang events inside the detector.

\section{A. Multiple-bang events}

If $L$ is similar or larger than the interaction length $L_{0}$, then the neutrino may interact more than once inside the detector. This is possible because the neutrino is not destroyed in the first eikonal interaction, it keeps going with basically the same energy and can interact again.

Let us assume that $\sigma_{\text {int }}^{\nu N} \approx \sigma_{\text {eik }}^{\nu N} \gg \sigma_{\mathrm{BH}}^{\nu N}, \sigma_{\mathrm{SM}}^{\nu N}$ and let us neglect the amount of energy lost by the neutrino in each interaction. It is straightforward to find the probability of having exactly $N$ interactions (bangs) in a length $L$ :

$$
P_{N}(L)=\mathrm{e}^{-L / L_{0}} \frac{\left(L / L_{0}\right)^{N}}{N !} .
$$

For example, the probability of having only one interaction would be $P_{1}(L)=\exp \left(-L / L_{0}\right)\left(L / L_{0}\right)$; for $L \ll L_{0}$ we have $P_{1}(L) \approx L / L_{0}$, but for $L \gg L_{0}$ this amplitude goes to zero (it is very unlikely to have only one interaction). Given $L$, the most probable number of interactions is $N=L / L_{0}$, which is also the average number of interactions:

$$
\langle N\rangle=\sum_{N=1}^{\infty} N P_{N}=\frac{L}{L_{0}} .
$$

Notice that the probability of having any type of event, i.e., at least one interaction, is (see Eq. (25))

$$
P(L)=\sum_{N=1}^{\infty} P_{N}=1-\mathrm{e}^{-L / L_{0}},
$$

whereas the probability that this event includes more than one interaction (a multiple-bang event) would be $P(L)-P_{1}(L)$ :

$$
P_{\text {mult }}(L)=1-\mathrm{e}^{-L / L_{0}}\left(1+L / L_{0}\right) .
$$

Double-bang events could also be produced by SM or $\mathrm{BH}$ interactions. Within the SM, the second bang would correspond to the decay of the tau created in the first interaction. The probability that this happens would be the probability that a $\nu_{\tau}$ has a SM charged current interaction times the probability that the tau lepton decays inside the detector. If the $\nu N$ interaction results into a $\mathrm{BH}$, its evaporation would also produce taus that could decay inside the detector. For the double-bang tau event to be contained inside a detector like IceCube $(1 \mathrm{~km}$ of length with $125 \mathrm{~m}$ between strings), the energy of the tau lepton must be between $2.5 \times 10^{6} \mathrm{GeV}$ and $10^{7} \mathrm{GeV}$ []].

\section{B. Numerical example}

Let us consider again a single neutrino of $E_{\nu}=10^{10}$ $\mathrm{GeV}$. Within the SM, its interaction length in ice is $L_{0}^{\mathrm{SM}}=440 \mathrm{~km}$. This means that typically it could reach the center of AMANDA or IceCube $(1.8 \mathrm{~km}$ below the antarctic ice 15]) from angles $\cos \theta_{z} \geq-0.03$. If there are $n=2(6)$ extra dimensions and $M_{D}=1 \mathrm{TeV}$, the interaction length before a hard gravitational interaction would be just $L_{0}=17 \mathrm{~km}(4 \mathrm{~km})$, which corresponds to $\cos \theta_{z} \geq 0.11(0.44)$. For $M_{D}=2.8(4.5) \mathrm{TeV} \sigma_{\mathrm{BH}}^{\nu N} \approx \sigma_{\mathrm{SM}}^{\nu N}$ and $L_{0} \approx L_{0}^{\mathrm{SM}}$.

If the neutrino reaches the detector, within the SM the probability that in $L=1 \mathrm{~km}$ (the longitudinal dimension of IceCube) it starts a hadronic shower is $P_{\text {int }}^{\mathrm{SM}}=2.2 \times$ $10^{-3}$. For a $\nu_{\tau}$ neutrino, the probability of an event with a tau lepton in the initial point of the hadronic shower is $1.6 \times 10^{-3}$.

If $M_{D}=1 \mathrm{TeV}$ the probability of a short distance gravitational event would be $P_{\mathrm{int}}^{\mathrm{BH}}=0.06$ if $n=2$ (or 0.22 for $n=6)$. To find the probability of a soft eikonalized interaction we need to evaluate

$$
\sigma_{\text {eik }}^{\nu N}=\int_{y_{\min }}^{y_{\max }} d y \frac{d \sigma_{\text {eik }}^{\nu N}}{d y}
$$

with $y_{\max }=0.2$ and $y_{\min }=(100 \mathrm{TeV}) / E_{\nu}$ (the energy of the shower should be above $100 \mathrm{TeV}$ to avoid the atmospheric background). The probability of an event in a length $L$ would then correspond to $\sigma_{\text {int }} \approx \sigma_{\text {eik }}^{\nu N}$ in Eq. (25), which for $L=1 \mathrm{~km}$ and $n=2$ (6) gives $P_{\text {int }}^{\text {eik }}=0.56(0.33)$. This probability includes events with one bang: $P_{1}^{\text {eik }}=0.36(0.27)$, with two bangs: $P_{2}^{\text {eik }}=0.15(0.06)$, and with more than two bangs: $P_{>2}^{\text {eik }}=0.05(0.008)$.

As explained above, double-bang events could also be produced by SM interactions. The probability that a $10^{10} \mathrm{GeV} \nu_{\tau}$ produces a tau lepton of energy between $2.5 \times 10^{6}$ and $10^{7} \mathrm{GeV}$ is just $P_{2}^{\mathrm{SM}} \approx 6.8 \times 10^{-5}$.

In Fig. 4 we express the probability $P_{\text {surv }}$ that the $10^{10}$ $\mathrm{GeV}$ neutrino survives through $1.8 \mathrm{~km}$ of ice to reach vertically IceCube for different values of $M_{D}$ (for large values of $M_{D} P_{\text {surv }} \approx P_{\text {surv }}^{\mathrm{SM}} \approx 1$ ). We also plot the probability that if it has reached the detector it experiences 

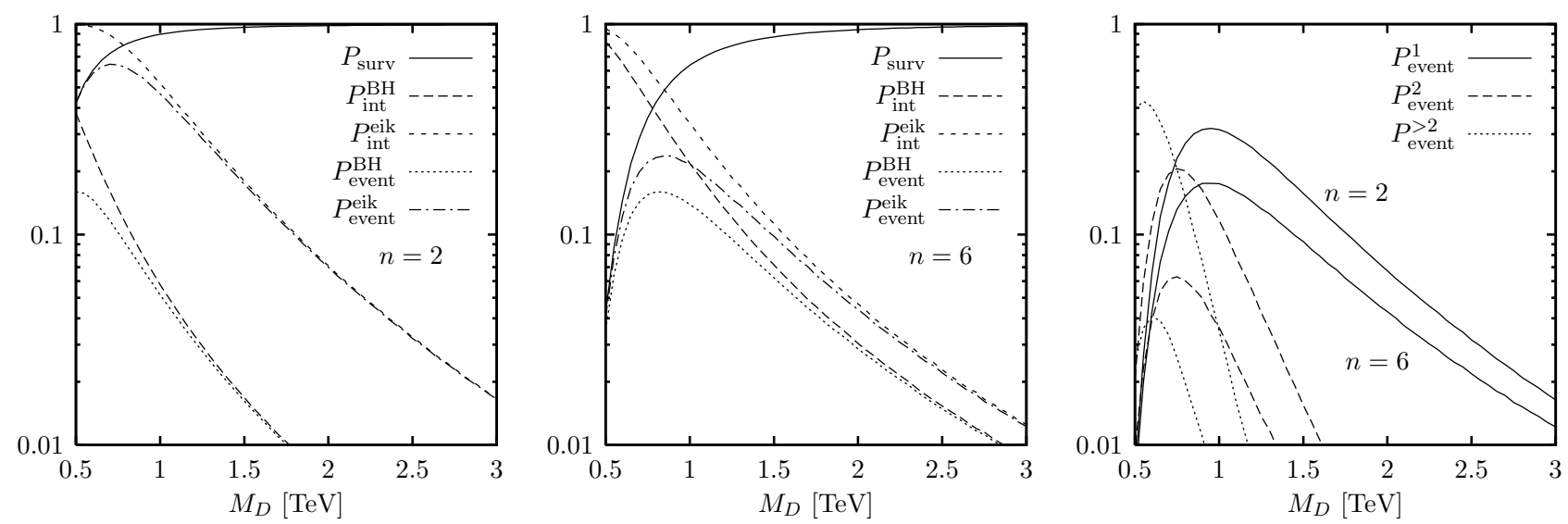

FIG. 4: The different probabilities defined in the text for a $10^{10} \mathrm{GeV}$ neutrino reaching IceCube from $\theta_{z}=0$ as a function of $M_{D}$ for $n=2$ and $n=6$.

a hard interaction $\left(P_{\mathrm{int}}^{\mathrm{BH}}\right)$ or a soft observable interaction $\left(P_{\mathrm{int}}^{\text {eik }}\right)$ within a distance of $L=1 \mathrm{~km}$. The product $P_{\text {event }}=P_{\text {surv }} P_{\text {int }}$ would give the probability that the neutrino gives a signal in IceCube. We obtain that this probability is larger for eikonal than for $\mathrm{BH}$ events. We also find that it is maximal for $M_{D} \approx 0.8 \mathrm{TeV}$. For lower values of $M_{D}$ the neutrino tends to interact before reaching the detector, and for larger values it tends to go through the detector without interactions. In Fig. 4 we also plot the probability of an eikonal event that includes only one bang $\left(P_{\text {event }}^{1}\right)$, two bangs $\left(P_{\text {event }}^{2}\right)$, or more than two bangs $\left(P_{\text {event }}^{>2}\right)$. If $M_{D} \gtrsim 1.5 \mathrm{TeV}$ the probability of more than one bang would be very small.

Within a distance $L$ the average energy lost by the neutrino in eikonal interactions and radiated through gravitational bremsstrahlung would be, respectively,

$$
\begin{gathered}
\left\langle E_{\text {eik }}\right\rangle=L \rho N_{A} \int_{0}^{y_{\max }} d y y E_{\nu} \frac{d \sigma_{\text {eik }}^{\nu N}}{d y}, \\
\left\langle E_{\mathrm{rad}}\right\rangle=L \rho N_{A} \int_{M_{D}^{2} / s}^{1} d x \int_{0}^{y_{\max }} d y E_{\mathrm{rad}} \frac{d^{2} \sigma_{\text {eik }}^{\nu N}}{d x d y},
\end{gathered}
$$

with $E_{\text {rad }}$ given in Eq. (20). For $L=1 \mathrm{~km}$ and $n=2(6)$ the $10^{10} \mathrm{GeV}$ neutrino will lose $\left\langle E_{\text {eik }}\right\rangle=6 \times 10^{7}(1.2 \times$ $\left.10^{8}\right) \mathrm{GeV}$ to hadrons and $\left\langle E_{\mathrm{rad}}\right\rangle=9.2 \times 10^{7}\left(1.2 \times 10^{8}\right)$ $\mathrm{GeV}$ to gravitational radiation. This means that, as it propagates in the detector, the energy loss in these soft processes is negligible. In a typical interaction length $L_{0}$ of a hard interaction (where the neutrino will lose most or all of its energy) we find that $\left\langle E_{\text {eik }}\right\rangle / E_{\nu}=0.10(0.05)$ and $\left\langle E_{\mathrm{rad}}\right\rangle / E_{\nu}=0.16(0.05)$.

Finally, let us study what is the typical energy of the hadronic shower started by the $10^{10} \mathrm{GeV}$ neutrino. We will increase $M_{D}$ to $2 \mathrm{TeV}$ (to avoid double-bang events) and take $n=2(6)$. If the neutrino reaches the detector at IceCube, the probability that it starts a shower of en-

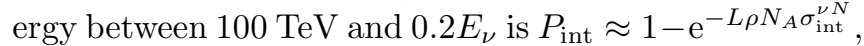
where $\sigma_{\text {int }}^{\nu N} \approx \sigma_{\text {eik }}^{\nu N}$ with $y_{\min }=100 \mathrm{TeV} / E_{\nu}$ and $y_{\max }=$

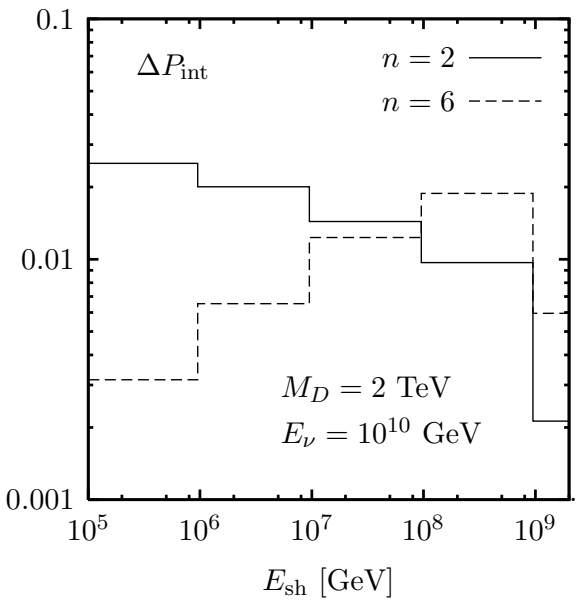

FIG. 5: Probability that a $10^{10} \mathrm{GeV}$ neutrino that reaches IceCube starts an eikonal shower in each interval of energies for $M_{D}=2 \mathrm{TeV}$ and $n=2,6$. For example, the neutrino has a probability of 0.024 of starting a shower of energy between 100 and $1000 \mathrm{TeV}$ if $n=2$.

0.2. We obtain $P_{\text {int }}=0.070(0.045)$. Now, the probability $\Delta P_{\text {int }}$ that this event has an energy between $E_{1}$ and $E_{2}$ is

$$
\Delta P_{\mathrm{int}}=\frac{P_{\mathrm{int}}}{\sigma_{\mathrm{eik}}^{\nu N}} \int_{\frac{E_{1}}{E_{\nu}}}^{\frac{E_{2}}{E_{\nu}}} d y \frac{d \sigma_{\mathrm{eik}}^{\nu N}}{d y} .
$$

In Fig. 5 we divide the interval from $100 \mathrm{TeV}$ to $2 \times 10^{9}$ $\mathrm{GeV}$ in 5 bins and express the probability that the shower energy is in each of the bins. We observe that the typical value is between $10^{5}$ and $10^{8} \mathrm{GeV}$ for $n=2$ and between $10^{6}$ and $10^{9} \mathrm{GeV}$ for $n=6$.

\section{Bounds from air showers}

The fact that the typical energy $E_{\mathrm{sh}}=y E_{\nu}$ of the shower started in these processes is much smaller than 
the energy $E_{\nu} \approx 10^{10} \mathrm{GeV}$ of the incoming neutrino has implications in air shower experiments. In particular, the absence of deeply penetrating showers in AGASA and Fly's Eye could exclude $\nu N$ cross sections between 0.01 and $1 \mathrm{mb}$. Notice, however, that these experiments are only sensitive to showers of very large energy, with $E_{\text {sh }}$ around or above $10^{9} \mathrm{GeV}$. Since the eikonal cross sections that we are considering reach a large size only for low values of $y$, the typical showers that they produce would be invisible in AGASA and Fly's Eye. This is in contrast to processes like $\mathrm{BH}$ formation 5| or other processes of strongly interacting neutrinos [25], where most of the energy of the initial neutrino would be transferred to the shower.

A precise analysis of the bounds on $M_{D}$ from eikonal processes in deep air shower experiments can be found in 12. The limits obtained there, between $1 \mathrm{TeV}$ for $n=2$ and $1.5 \mathrm{TeV}$ for $n=6$, are essentially the same as the ones from $\mathrm{BH}$ production in [5].

\section{Cosmogenic neutrinos at AMANDA and IceCube}

Let us now study the total number of events at AMANDA $\left(0.03 \mathrm{~km}^{2}\right.$ and a length of $\left.700 \mathrm{~m}\right)$ and IceCube $\left(1 \mathrm{~km}^{3}\right)$ for the neutrino fluxes in Fig. 3

In the SM, for the higher flux (910 downward cosmogenic neutrinos of each flavor per year and $\mathrm{km}^{2}$ ), we would expect 1.32 contained events per year in IceCube. Of those, 0.38 would come from a neutral current and 0.94 from a charged current (one third of the events of each lepton flavor). The distribution of energy of these events is given in Fig. [6] (we show it despite the low statistics just for a comparison purpose). Around 0.008 of the 0.31 tau events would decay and give double bangs inside the detector. For the lower flux (around 410 cosmogenic neutrinos of each flavor per year and $\mathrm{km}^{2}$ ), we would expect just $0.50 \mathrm{SM}$ events per year inside the detector, 0.12 of them containing a tau lepton, and just 0.003 double-bang events. The numbers for AMANDA can be easily obtained just multiplying by a volume factor $V_{A M} / V_{I C} \approx 0.02$, namely $0.03 \mathrm{SM}$ events per year for the higher flux and 0.01 for the lower flux.

In a scenario with $n=2(6)$ extra dimensions, for the higher flux we obtain a signal above the SM background (1.32 contained events per year in IceCube) if $M_{D} \leq$ $5.6(4.9) \mathrm{TeV}$, whereas for the lower flux we have a signal above the $0.50 \mathrm{SM}$ events if $M_{D} \leq 4.8(4.5) \mathrm{TeV}$.

The event rate at IceCube and AMANDA for different values of $M_{D}$ and a minimum energy of the shower of $100 \mathrm{TeV}$ is plotted in Fig. 7 We give the number of short distance (BH) and of soft (eikonal) events. We also include the number of double-bang eikonal events, which is significant for low values of $M_{D}$.

The energy distributions of contained hadronic showers in IceCube for both fluxes, $M_{D}=2 \mathrm{TeV}$ and $n=2(6)$ are also shown in Fig. 6] There is a clear difference be-

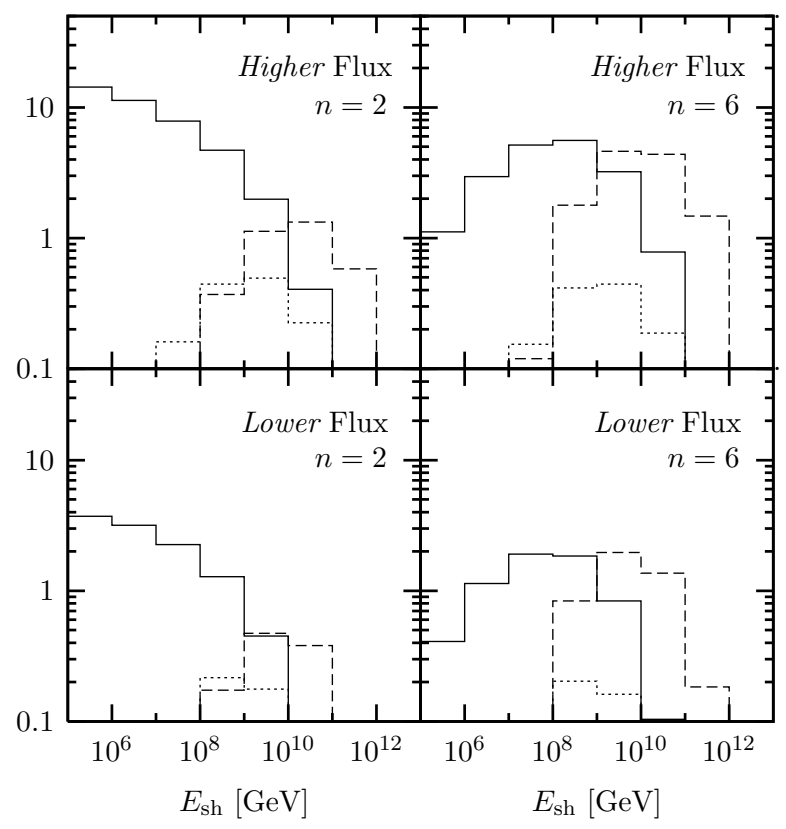

FIG. 6: Energy distribution (events per bin) of the eikonal (solid), BH (dashed) and SM (dotted) events in IceCube per year for the higher and the lower cosmogenic fluxes, $M_{D}=$ $2 \mathrm{TeV}$ and $n=2,6$.

tween the energy distribution of eikonal and $\mathrm{BH}$ or SM events: while these have a shape similar to the cosmogenic flux, eikonal events are typically of much lower energies, specially for $n=2$.

\section{SUMMARY AND DISCUSSION}

Cosmogenic neutrinos interact with terrestrial nucleons at center of mass energies $\sqrt{2 m_{N} E_{\nu}} \approx 100 \mathrm{TeV}$, so they can be used as probes of new $\mathrm{TeV}$ physics in neutrino telescopes. In particular, the possibility of $\mathrm{BH}$ formation in models with extra dimensions has been entertained by several groups. These analyses are based on a geometric cross section that assumes single $\mathrm{BH}$ production whenever the neutrino and the parton interact at impact parameters smaller than $R_{S}$. The problem with this estimate is that, despite the large energy of cosmogenic neutrinos, the $\nu N$ cross section is dominated by the small $x$ region and most of the BHs produced in a neutrino telescope would be very light, with masses just above $M_{D}$. These light BHs would be very sensitive to effects like graviton emission during the collapse or nonthermal effects in the evaporation, which add uncertainty to any estimate.

In this paper we have analyzed in detail a different type of signal. It is produced when the neutrino interacts elastically with a parton at typical distances larger than $R_{S}$ and transfers a small fraction $y$ of its energy. The process is properly described by the eikonal approximation. We have shown that the cutoff dependence of the eikonal 


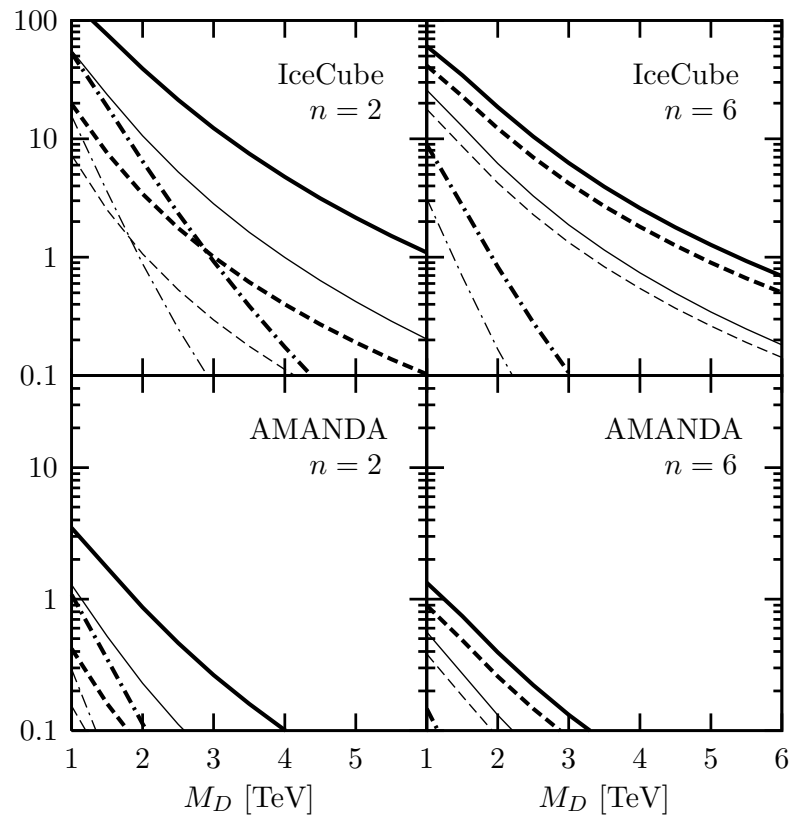

FIG. 7: Contained events per year in IceCube and AMANDA for the higher (thick) and the lower (thin) cosmogenic fluxes and $n=2,6$. We show eikonal (solid), multi-bang (dasheddotted) and $\mathrm{BH}$ (dashed) events.

cross section is small (see Fig. 2), and that non-linear corrections and graviton emission are negligible. The distinct experimental signature of these processes would be a hadronic shower of energy $y E_{\nu} \ll E_{\nu}$. A muon, a tau, or an electromagnetic shower would never be produced in the initial $\nu N$ interaction.

If IceCube observes contained showers above $100 \mathrm{TeV}$, their energy distribution and the absence of charged leptons in the starting point of the shower would then suffice to decide whether they are due to SM or TeV-gravity interactions, as we argue below.

Let us first suppose that the cosmogenic neutrino flux (important at energies between $10^{8}$ and $10^{11} \mathrm{GeV}$ ) is smaller than expected (see the minimal flux in Fig. B3). In this case there will be no SM contained showers in IceCube. However, for sufficiently low values of $M_{D}$ (the optimal value is $0.8 \mathrm{TeV}$, see $P_{\text {event }}^{\text {eik }}$ in Fig. [6) the eikonal cross section grows and even a very low cosmogenic flux could imply contained showers from gravitational interactions. These showers would be typically less energetic than the initial neutrino that produce them (see distribution in Fig. (6). One may then wonder if these events could be due to a large flux of neutrinos in the intermediate energy range $\left(10^{5}-10^{8} \mathrm{GeV}\right)$. The observation of an initial muon in a $24 \%$ of the showers would be characteristic of SM interactions in this range of energies, whereas the absence of muon events would be consistent with soft gravitational interactions of cosmogenic neutrinos of much higher energy. In addition, the SM contained showers would always come together with a large number of muon events of similar energy produced outside the de- tector, which would be absent for TeV-gravity contained showers.

Let us now suppose a flux of cosmogenic neutrinos within the expected limits (fluxes higher and lower in Fig. 31). In this case IceCube will observe SM contained showers in the range $10^{8}$ to $10^{11} \mathrm{GeV}$. If $M_{D} \approx 5 \mathrm{TeV}$ the number of eikonal processes will be similar to the number of SM events. However, their energy distribution will be different and both types of processes can be separated. Again, the absence of charged leptons in the initial interaction point would distinguish these events from SM events of lower energies. For the higher flux in Fig. 3 we expect more than one contained non-standard shower per year in IceCube if $M_{D} \leq 6.0(5.5) \mathrm{GeV}$ for $n=2(6)$.

We then conclude that for any intermediate-energy and cosmogenic neutrino fluxes, an enhanced rate of neutral versus charged current events of energies above $100 \mathrm{TeV}$ could be explained by $\mathrm{TeV}$-gravity interactions.

These interactions could also produce a very peculiar signal for relatively low values of $M_{D}$. In a typical eikonal process the neutrino loses a small fraction $y$ of its energy and keeps going, so it can interact several times inside the detector. This effect is specially important for low values of $n$, where gravity dilutes slowly with the distance (notice that for $n<2$ it becomes a long-distance interaction and the total eikonal cross section is divergent). If $n=2$ and $M_{D} \leq 0.9 \mathrm{TeV}$ the average interaction length of a $10^{10} \mathrm{GeV}$ neutrino between two eikonal interactions of $E>100 \mathrm{TeV}$ becomes smaller than the longitudinal dimension of IceCube. Therefore, we would expect two (or more) bangs of $10^{5}$ to $10^{8} \mathrm{GeV}$ inside the detector. We think this type of events could be easily distinguished from possible double-bang SM events, where the first bang corresponds to a $\nu_{\tau} N$ charged current interaction and the second one to a tau decay. First of all, the typical energy of the SM double-bang event would be necessarily between $2.5 \times 10^{6}$ and $10^{7} \mathrm{GeV}$; for lower energies the tau decays before $125 \mathrm{~m}$ (the separation between strings at IceCube) and for larger energies the second bang would be out of the detector. Second, there should be a clear trace in the detector as the tau propagates between the two bangs, which is absent in TeV-gravity events.

In summary, we think that elastic eikonalized interactions provide a clear (distinguishable from possible SM events) and model-independent (insensitive to how the theory is completed in the UV) signal of TeV gravity. Being at impact parameter distances larger than $R_{S}$, these interactions have a cross section that is larger than the geometric cross section to produce a BH. The eikonal event would be much less energetic than a $\mathrm{SM}$ or a $\mathrm{BH}$ event, but neutrino telescopes are sensitive to showers of energy up to four orders of magnitude below the average energy of cosmogenic neutrinos. The values of the fundamental scale of gravity that IceCube could reach, around $5 \mathrm{TeV}$, are comparable to those to be explored at the LHC or the ILC [14].

This work has been supported by MCYT (FPA2003- 
09298-C02-01) and Junta de Andalucía (FQM-101). J.I.I. and D.M. acknowledge financial support from the European Community's Human Potential Programme HPRN-CT-2000-00149. We thank Eduardo Battaner,
Tommaso Chiarusi, Francis Halzen, Marek Kowalski, Paolo Lipari, Teresa Montaruli, Sergio Navas, Andreas Ringwald and Christian Spiering for useful discussions.
[1] L. Anchordoqui, T. Paul, S. Reucroft and J. Swain, Int. J. Mod. Phys. A 18 (2003) 2229.

[2] D. V. Semikoz and G. Sigl, JCAP 0404 (2004) 003.

[3] N. Arkani-Hamed, S. Dimopoulos and G. Dvali, Phys. Lett. B 429 (1998) 263; I. Antoniadis, N. Arkani-Hamed, S. Dimopoulos and G. Dvali, Phys. Lett. B 436 (1998) 257.

[4] P. C. Argyres, S. Dimopoulos and J. MarchRussell, Phys. Lett. B 441 (1998) 96; R. Emparan, G. T. Horowitz and R. C. Myers, Phys. Rev. Lett. 85 (2000) 499; D. M. Eardley and S. B. Giddings, Phys. Rev. D 66 (2002) 044011. S. Dimopoulos and G. Landsberg, Phys. Rev. Lett. 87 (2001) 161602; S. B. Giddings and S. Thomas, Phys. Rev. D 65 (2002) 056010.

[5] J. L. Feng and A. D. Shapere, Phys. Rev. Lett. 88 (2002) 021303; L. A. Anchordoqui, J. L. Feng, H. Goldberg and A. D. Shapere, Phys. Rev. D 65 (2002) 124027; Phys. Rev. D 66 (2002) 103002; Phys. Rev. D 68 (2003) 104025.

[6] A. Ringwald and H. Tu, Phys. Lett. B 525 (2002) 135; M. Kowalski, A. Ringwald and H. Tu, Phys. Lett. B 529 (2002) 1; S. I. Dutta, M. H. Reno and I. Sarcevic, Phys. Rev. D 66 (2002) 033002; A. Cafarella, C. Coriano and T. N. Tomaras, "Cosmic ray signals from mini black holes in models with extra dimensions: An analytical / Monte Carlo study", arXiv:hep-ph/0410358.

[7] J. Álvarez-Muñiz, J. L. Feng, F. Halzen, T. Han and D. Hooper, Phys. Rev. D 65 (2002) 124015.

[8] E. J. Ahn, M. Cavaglia and A. V. Olinto, arXiv:hep-ph/0312249

[9] R. Casadio and B. Harms, Int. J. Mod. Phys. A 17 (2002) 4635; D. Stojkovic, Phys. Rev. Lett. 94 (2005) 011603; T. G. Rizzo, "Collider production of $\mathrm{TeV}$ scale black holes and higher-curvature gravity", arXiv:hep-ph/0503163 J. L. Hewett, B. Lillie and T. G. Rizzo, "Black holes in many dimensions at the LHC: Testing critical string theory", arXiv:hep-ph/0503178 H. Yoshino and V. S. Rychkov, "Improved analysis of black hole formation in highenergy particle collisions", arXiv:hep-th/0503171

[10] S. B. Giddings and V. S. Rychkov, Phys. Rev. D 70 (2004) 104026.

[11] R. Emparan, M. Masip and R. Rattazzi, Phys. Rev. D 65 (2002) 064023; M. Masip, arXiv:hep-ph/0210143
[12] J. I. Illana, M. Masip and D. Meloni, Phys. Rev. Lett. 93 (2004) 151102; D. Meloni, Acta Phys. Polon. B 35 (2004) 2781.

[13] G. 't Hooft, Phys. Lett. B 198 (1987) 61; I. J. Muzinich and M. Soldate, Phys. Rev. D 37 (1988) 359; D. Amati, M. Ciafaloni and G. Veneziano, Phys. Lett. B 197 (1987) 81; D. Kabat and M. Ortiz, Nucl. Phys. B 388 (1992) 570.

[14] G. F. Giudice, R. Rattazzi and J. D. Wells, Nucl. Phys. B 630 (2002) 293.

[15] J. Ahrens [IceCube Collaboration], arXiv:astro-ph/0305196 see also http://icecube.wis.edu/

[16] E. A. Mirabelli, M. Perelstein and M. E. Peskin, Phys. Rev. Lett. 82 (1999) 2236; G. F. Giudice, R. Rattazzi and J. D. Wells, Nucl. Phys. B 544 (1999) 3; for a review, see F. Feruglio, arXiv:hep-ph/0401033

[17] S. Cullen and M. Perelstein, Phys. Rev. Lett. 83 (1999) 268; S. Hannestad and G. G. Raffelt, Phys. Rev. D 67 (2003) 125008 [Erratum-ibid. D 69 (2004) 029901].

[18] G. F. Giudice, T. Plehn and A. Strumia, Nucl. Phys. B 706 (2005) 455.

[19] S. Cullen, M. Perelstein and M. E. Peskin, Phys. Rev. D 62 (2000) 055012; F. Cornet, J. I. Illana and M. Masip, Phys. Rev. Lett. 86 (2001) 4235.

[20] I. Antoniadis and K. Benakli, Phys. Lett. B 326 (1994) 69; S. A. Abel, M. Masip and J. Santiago, JHEP 0304 (2003) 057.

[21] D. Amati, M. Ciafaloni and G. Veneziano, Nucl. Phys. B 347 (1990) 550.

[22] H. L. Lai et al. [CTEQ Collaboration], Eur. Phys. J. C 12 (2000) 375.

[23] P. Sreekumar et al., Astrophys. J. 494 (1998) 523.

[24] Z. Fodor, S. D. Katz, A. Ringwald and H. Tu, JCAP 0311 (2003) 015.

[25] G. Domokos and S. Kovesi-Domokos, Phys. Rev. Lett. 82 (1999) 1366; W. S. Burgett, G. Domokos and S. KovesiDomokos, "Low scale string unification and the highest energy cosmic rays", arXiv:hep-ph/0209162 Z. Fodor, S. D. Katz, A. Ringwald and H. Tu, "Strongly interacting neutrinos as the highest energy cosmic rays", arXiv:hep-ph/0310112 